\title{
Control alternativo de Aethina tumida Murray (Coleoptera: Nitidulidae) con polvos vegetales
}

\author{
Alternative control of Aethina tumida Murray (Coleoptera: Nitidulidae) with botanical dusts
}

\author{
Jorge Ismael Tucuch-Haas ${ }^{1}$ (D), María Alma Rangel-Fajardo ${ }^{1}$ (D) Fernando Casanova-Lugo ${ }^{2}$ (iD) \\ Esaú Ruíz-Sánchez ${ }^{3}$ iD, Fernando Utrera-Quintana ${ }^{4}$ (D) Cesar Jacier Tucuch-Haas ${ }^{5 *}$ (iD
}

\author{
${ }^{1}$ Campo Experimental Mocochá, Instituto Nacional de Investigaciones Forestales, Agrícolas y Pecuarias, \\ km 25 antigua carretera Mérida-Motul, 97454, Mocochá, Yucatán, México. \\ ${ }^{2}$ Tecnologico Nacional de México/Instituto Tecnológico de la Zona Maya, Carretera Chetumal-Escárcega Km 21.5, \\ Ejido Juan Sarabia, 77965, Othón P. Blanco, Quintana Roo, México. \\ ${ }^{3}$ Tecnológico Nacional de México/Instituto Tecnológico de Conkal, Avenida Tecnológico s/n, 97345, Conkal, Yucatán, México. \\ ${ }^{4}$ Facultad de Medicina Veterinaria y Zootecnia, Benemérita Universidad Autónoma de Puebla, \\ Carretera Cañada Morelos km 7.5, 75492, El Salado Tecamachalco, Puebla, México. \\ ${ }^{5}$ Tecnológico Nacional de México/ITS del Sur del Estado de Yucatán, carretera Muna-Felipe Carrillo Puerto \\ Tramo Oxkutzcab-Akíl, km. 41+400, 97880, Oxkutzcab, Yucatán, México. \\ *Autor para correspondencia: cesar_5204@hotmail.com
}

Fecha de recepción:

4 de junio de 2020

Fecha de aceptación:

8 de julio de 2020

Disponible en línea:

23 de octubre de 2020

Este es un artículo en acceso abierto que se distribuye de acuerdo a los términos de la licencia Creative Commons.

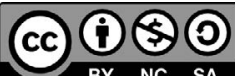

Reconocimiento-

NoComercial-

CompartirIgual 4.0

Internacional

\section{RESUMEN}

Aethina tumida Murray (Coleoptera: Nitidulidae), es una plaga de Apis mellifera L. (Hymenoptera: Apidae), responsable de pérdidas económicas en la producción de miel. En México no existe un producto autorizado para su control. El objetivo principal de esta investigación fue evaluar el efecto tóxico de Dysphania ambrosioides L., Ocimum basilicum L. y Origanum vulgare L. en la sobrevivencia de adultos de $A$. tumida. Dysphania ambrosioides registró la mayor tasa de mortalidad (100\%) a 1, 5 y $10 \mathrm{~g}$ después de 24 o 48 h del tratamiento, siendo estadísticamente diferente al resto de los tratamientos ( $\mathrm{P} \leq 0.05)$. Para O. basilicum y O. vulgare, la dosis y el tiempo jugaron un papel importante en su efectividad (69.2 y 65.8\% respectivamente) con la dosis de $10 \mathrm{~g}$ a las $48 \mathrm{~h}$ posteriores al tratamiento. El polvo de D. ambrosioides fue el mejor tratamiento para el control de A. tumida.

\section{PALABRAS CLAVE}

Dysphania ambrosioides, Ocimum basilicum, Origanum vulgare, Apis mellifera, Apicultura.

\begin{abstract}
Aethina tumida Murray (Coleoptera: Nitidulidae), is a pest of Apis mellifera L. (Hymenoptera: Apidae), responsible for economic losses in honey production. In Mexico, there are no authorized pesticide products for its control. The main objective of this investigation was to evaluate the toxic effect of Dysphania ambrosioides L., Ocimum basilicum L., and Origanum vulgare L. on the survival of adults of $A$. tumida. Dysphania ambrosioides registered the highest mortality rate (100\%) at 1,5 and $10 \mathrm{~g} 24$ or 48 hours after treatment, being statistically different from the rest of the treatments $(\mathrm{P} \leq 0.05)$. Treatments based on $O$. basilicum and O. vulgare, in which dose and time played an important role, both demonstrated greater effectiveness (69.2 and 65.8\% respectively), with a 10 $\mathrm{g}$ dose 48 hours after treatment. Dysphania ambrosioides powder proved to be the best treatment for the control of A. tumida.
\end{abstract}

\section{KEYWORDS}

Dysphania ambrosioides, Ocimum basilicum, Origanum vulgare, Apis mellifera, apiculture. 


\section{INTRODUCCIÓN}

La abeja, Apis mellifera L. (Hymenoptera: Apidae), es una especie de importancia mundial por sus múltiples beneficios, como: la producción de miel, la polinización y la conservación de las especies (May 2015). Para México, la apicultura es relevante por sus aportes socioeconómicos y ecológicos (Magaña et al. 2017), ya que genera divisas (56.4 millones de dólares) con la producción de miel, polen y otros productos de la colmena (FAO 2010). Además, al recolectar el néctar y el polen de las flores, las abejas fomentan la polinización, con lo cual contribuyen a la multiplicación y conservación de las especies (Sosenski y Domínguez 2018).

Por lo anterior, resulta de suma importancia cuidar y proteger las colonias de A. mellifera; sin embargo, la actividad apícola se ve afectada por plagas y enfermedades, entre las cuales se encuentran Varroa destructor (Anderson y Trueman), Nosema apis (Zander), Acarapis woodi (Rennie) (Medina-Flores et al. 2014) y recientemente Aethina tumida (Murray), el pequeño escarabajo de la colmena (PEC) (Al Toufailia et al. 2017).

Aethina tumida es un parásito-carroñero de $A$. mellifera y otras colonias de abejas sociales originario del África subsahariana, donde representa una plaga menor (Neumann et al. 2016). Ha sido reportado en Italia (Palmeri et al. 2015), Brasil (Pereira et al. 2019), Costa Rica (Calderón y Ramírez 2019), Estados Unidos, Canadá, México, Cuba y Nicaragua (Al Toufailia et al. 2017), lugares donde ha ocasionado la pérdida total de colonias. Los adultos de A. tumida se alimentan y desarrollan en el interior de la colmena; las hembras ovipositan en rendijas y cavidades, de donde eclosionarán las larvas que se alimentan de la cría de las abejas, así como de miel, polen y cera; todo ello, además de ocasionar la muerte de las crías, provoca la fermentación de la miel y la destrucción de los panales (Neumann et al. 2016).

Durante la alimentación de las larvas y los adultos de $A$. tumida, éstos defecan sobre los panales, ocasionando la fermentación de la miel y la dispersión de la colonia, para concluir con la enjambrazón o colapso de la colonia (Pirk et al. 2016). Cuando las larvas alcanzan el tamaño adecuado $(4.5 \mathrm{~mm}$ a $1.2 \mathrm{~cm}$ de largo y $1.6 \mathrm{~mm}$ de ancho) y están próximas a pupar, abandonan la colmena y se dejan caer para enterrarse y continuar con la metamorfosis que da origen a un escarabajo (Lundie 1940).

A pesar de los esfuerzos efectuados, actualmente, en México, no existe un producto registrado y autorizado por la Comisión Federal para la Protección Contra Riesgos Sanitarios (COFEPRIS 2019) para el control de A. tumida, que sea ecológico y libre de residuos químicos. Debido a lo antes mencionado, es de suma importancia desarrollar un método de control amigable con el ambiente, así como con el ser humano y con las abejas. Publicaciones recientes sostienen que extractos vegetales a base de epazote, orégano y albahaca poseen propiedades entomotóxicas para coleópteros; dichas plantas se encuentran disponibles en la Península de Yucatán (Rodríguez-González et al. 2019). Por lo tanto, la aplicación de polvos vegetales podría ser un método de control alternativo deseable. Con base en lo expuesto, el objetivo de esta investigación fue evaluar el efecto tóxico del epazote (Dysphania ambrosioides L.), el orégano (Origanum vulgare L.) y la albahaca (Ocimum basilicum L.) en la mortalidad del pequeño escarabajo de la colmena.

\section{Materiales y Métodos}

El estudio se llevó a cabo en el laboratorio de entomología del Instituto Nacional de Investigaciones Forestales, Agrícolas y Pecuarias, CIR-Sureste (Km. 25 Carretera Mérida-Motúl, Mocochá, Yucatán).

\section{Colonia de insectos}

Se estableció una colonia de A. tumida susceptible a insecticidas en cámaras de cría, con adultos (machos y hembras) sanos, originalmente recolectados en enjambres y colonias silvestres, donde se ha reproducido desde 2018 (20 \pm 4 generaciones). Las cámaras de cría consistieron en dos secciones, diseñadas con recipientes de plástico con capacidad de $5 \mathrm{~L}$; la primera sección - denominada superior o área de alimentación y apareamiento- contenía orificios (2 $\mathrm{mm}$ ) en la base del fondo, para permitir el traslado de la larva en etapa errante o próximo a pupar a la sección inferior (suelo). En la tapa de la sección superior se confeccionó una ventana (10 cm de diámetro), cubierta con tela de organza (-1 mm), para facilitar el flujo de aire (ventilación); en el interior de la sección superior, 
se introdujo cartón (20 cm de ancho y $15 \mathrm{~cm}$ de largo), cera estampada $\left(10 \mathrm{~cm}^{2}\right)$, sustituto de polen y miel (2:1 $\mathrm{p} / \mathrm{p}$ ) como fuente de alimento, proporcionado cada 7 días a razón de $10 \mathrm{~g}$ por cámara. Las condiciones de esta sección fueron $28 \pm 2^{\circ} \mathrm{C}$ y $70 \%$ humedad relativa. $\mathrm{La}$ segunda sección - denominada inferior o área de metamorfosis - contenía $300 \mathrm{~g}$ de suelo franco-arcilloso; a esto se le agregaron $10 \mathrm{~mL}$ de agua por semana para mantener la humedad y la temperatura a $28 \pm 2{ }^{\circ} \mathrm{C}$ en oscuridad permanente.

Una vez identificada la presencia de las primeras pupas, el sustrato de la parte inferior de la cámara se trasladó a un recipiente con $20 \mathrm{~L}$ de capacidad, en donde se esperó la emergencia de los adultos, para ser trasladados a otro recipiente de $2 \mathrm{~L}$ de capacidad y proporcionarles alimentación y cuidado, como lo proponen Mürrle y Neumann (2004) y Neumann et al. (2013). Para los ensayos se utilizaron escarabajos con 7 días de emergencia (de color oscuro o completamente esclerosado). El monitoreo y recolección de los escarabajos recién emergidos se hizo cada $24 \mathrm{~h}$.

\section{Material vegetal}

En los patios del municipio de Mocochá (Latitud $21^{\circ}$ $08^{\prime} \mathrm{N}$ y Longitud $89^{\circ} 27^{\prime} \mathrm{O}$ ), Conkal (Latitud $21^{\circ} 04^{\prime}$ $\mathrm{N}$ y Longitud $89^{\circ} 31^{\prime} \mathrm{O}$ ) y Mérida (Latitud $20^{\circ} 58^{\prime} \mathrm{N}$ y Longitud $89^{\circ} 37^{\prime} \mathrm{O}$ ), se colectaron cinco $\mathrm{kg}$ de hojas jóvenes de epazote (D. ambrosioides), albahaca $(O$. basilicum) y orégano(O.vulgare), provenientes de plantas de traspatio. Las hojas recién colectadas se enjuagaron con agua destilada y se colocaron sobre mesas de trabajo (expuestas al sol durante $24 \mathrm{~h}$ ), para su secado a temperatura ambiente $\left(33 \pm 35^{\circ} \mathrm{C}\right)$; posteriormente, se ingresaron al laboratorio, donde se colocaron en charolas de aluminio y se introdujeron dentro de un horno (BLUE M, modelo 0V-490A-2, graduado a una temperatura de $60^{\circ} \mathrm{C}$ constante), para eliminar la humedad ambiental, con revisiones periódicas cada 30 minutos hasta que la parte vegetativa alcanzó peso constante. Una vez seco el material vegetal, se procedió a pulverizarlo con un molino eléctrico (IKA Works, inc., modelo M20 S3) y se pasó por un tamiz con malla fina (0.841 mm); se obtuvieron $2 \pm 0.5 \mathrm{~kg}$ de polvo fino de cada material. Los polvos vegetales se almacenaron en frascos con cierre hermético, previamente etiquetados, y se conservaron a temperatura ambiente hasta su uso.

\section{Ensayo de mortalidad}

Se evaluaron tres polvos vegetales (D. ambrosioides, $O$. basilicum y O. vulgare), cada uno con cuatro niveles $(0,1$, 5 y 10 g), suministrado mediante contacto directo. Para tal propósito, se emplearon cajas Petri de plástico $(4 \mathrm{~cm}$ de diámetro), con ventilación, donde se introdujeron 10 escarabajos (previamente anestesiados con $\mathrm{CO}_{2}$ a 30 psi durante un minuto para facilitar su manipulación), a los que se les aplicó el polvo vegetal en la dosis correspondiente sobre el cuerpo del insecto (la cantidad aplicada se dejó en la caja Petri para el libre contacto polvo-insecto). Se consideró muerto aquel individuo que no moviera las antenas, que no caminara o no respondiera al toque del pincel (Tucuch-Haas et al. 2010). El máximo nivel de mortalidad aceptable para el testigo sin tratar fue $20 \%$ y se corrigió mediante la fórmula de Abbott (Abbott 1925). En todos los casos, los escarabajos tratados se mantuvieron a temperatura ambiente $\left(30 \pm 3{ }^{\circ} \mathrm{C}\right)$ y oscuridad total. La variable respuesta fue el porcentaje de mortalidad de los escarabajos (24 y $48 \mathrm{~h}$ después de iniciado el ensayo), que se calculó utilizando la fórmula de Baba (Baba-Tierto 1994).

\section{Diseño experimental y análisis estadístico}

Los experimentos fueron dispuestos bajo un diseño experimental completamente al azar, con arreglo factorial $3 \times 4 \times 2$, con 12 repeticiones; el factor principal fueron los polvos vegetales delas tresespecies en estudio, seguido de las cuatro dosis empleadas y dos tiempos de exposición; como unidad experimental se tomó una caja Petri con 10 individuos. Posteriormente, los datos obtenidos fueron analizados estadísticamente mediante el análisis de varianza (ANOVA); cuando se detectaron diferencias significativas, se aplicó la prueba de comparación de medias por Tukey, con un nivel de confianza de 95\%, usando el paquete SAS versión 9.3 (SAS 2011).

\section{RESULTADOS Y DISCUSIÓN}

\section{Mortalidad de A. tumida}

Los polvos evaluados a base de epazote (D. ambrosioides), albahaca (O. basilicum) y orégano (O. 
vulgare) demostraron tener efectos tóxicos sobre $A$. tumida (78.4, 47.1 y $42.2 \%$ respectivamente), lo cual apoya los resultados de Vite-Vallejo et al. (2018), quienes señalaron que algunos extractos a base de plantas (Apicure ${ }^{\circledR}$ ) poseen propiedades tóxicas sobre A. tumida. Además, son coincidentes con los resultados encontrados en otros estudios para plagas diferentes, como D. ambrosioides, que presentó actividad insecticida contra el escarabajo oscuro de las granjas avícolas Alphitobius diaperinus Panzer (Coleoptera: Tenebrionidae) (Arena et al. 2018); O. basilicum, el cual mostró toxicidad contra Spodoptera frugiperda J.E. Smith (Lepidoptera: Noctuidae) (Silva et al. 2017) y $O$. vulgare, que presenta metabolitos secundarios con actividad insecticida contra Bemisia tabaci Gennadius (Hemiptera: Aleyrodidae) (Vite-Vallejo et al. 2018); estos resultados los muestran como una herramienta para el manejo de plagas.

Cuadro 1. Ingredientes y composición química de las dietas experimentales $(\%)$

\begin{tabular}{|c|c|}
\hline Factor & $\begin{array}{c}\text { Variable respuesta } \\
\text { Mortalidad (\%) }\end{array}$ \\
\hline \multicolumn{2}{|l|}{ Control (especie vegetal) } \\
\hline O. vulgare & $42.28 \pm 3.20 \mathrm{~b}^{*}$ \\
\hline D. ambrosioides & $78.44 \pm 3.28$ a \\
\hline O. basilicum & $47.19 \pm 3.30 \mathrm{~b}$ \\
\hline Dms & 5.62 \\
\hline \multicolumn{2}{|l|}{ Dosis (gramos de polvo) } \\
\hline 0 & $8.89 \pm 1.64 \mathrm{c}$ \\
\hline 1 & $65.64 \pm 3.63 \mathrm{~b}$ \\
\hline 5 & $71.01 \pm 3.55 \mathrm{~b}$ \\
\hline 10 & $78.33 \pm 3.24 \mathrm{a}$ \\
\hline Dms & 7.12 \\
\hline \multicolumn{2}{|l|}{$\begin{array}{l}\text { Tiempo de exposición } \\
\text { (horas) }\end{array}$} \\
\hline 24 & $41.18 \pm 2.95 \mathrm{~b}$ \\
\hline 48 & $70.76 \pm 2.51 \mathrm{a}$ \\
\hline DMS & 3.84 \\
\hline
\end{tabular}

* = Medias con la misma letra en cada variable y cada factor no son estadísticamente diferentes (Tukey, $\mathrm{P} \leq 0.05$ ). DMS: diferencia mínima significativa.

La aplicación de los polvos vegetales evaluados mostró diferencias en la mortalidad de los escarabajos adultos $(\mathrm{P} \leq 0.05)$ (Cuadro 1). El tratamiento a base de D. ambrosioides fue el que arrojó la mejor respuesta, con
$78.4 \%$ de mortalidad en promedio, después de las $48 \mathrm{~h}$ de haber sido aplicado el tratamiento; estadísticamente, fue el más diferente al resto de los tratamientos ( $\mathrm{P} \leq$ 0.05). El resto de los extractos vegetales registraron una mortalidad de 47.1 y $42.2 \%$, respectivamente, para $O$. basilicum y $O$. vulgare, lo cual representa un efecto de 40 y $46.5 \%$ por debajo del extracto de epazote. La dosis de $10 \mathrm{~g}$ demostró $78 \%$ de mortalidad y una diferencia significativa con respecto a la dosis de $0 \mathrm{~g}$, es decir, $50 \%$ de diferencia (Cuadro 1). Por su parte, para el tiempo de exposición, el lapso de $48 \mathrm{~h}$ presentó el máximo porcentaje de mortalidad con $29.58 \%$ por encima del de $24 \mathrm{~h}$ (Cuadro 1).

En la interacción dosis-especie, se puede apreciar una diferencia $(\mathrm{P} \leq 0.05)$ entre las diferentes dosis y el testigo, mientras que entre las dosis (1, 5 y 10 g), sólo D. ambrosioides no presentó diferencias significativas; se reportó $100 \%$ de mortalidad para las tres concentraciones, contrario a lo ocurrido para O. vulgare y $O$. basilicum, en los cuales la efectividad estuvo en función de la dosis: a mayor dosis mayor efectividad (Figura 1).

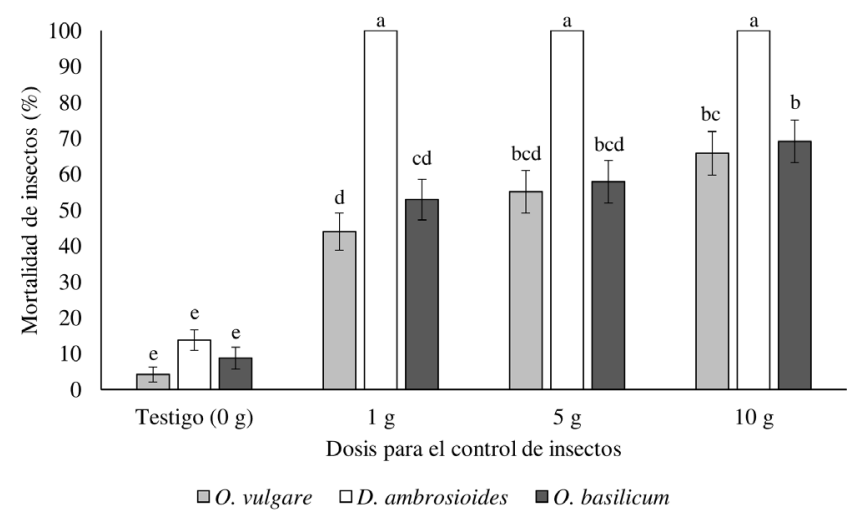

Figura 1. Mortalidad de insectos adultos de Aethina tumida tratados con diferentes polvos de tres especies de plantas colectadas en Yucatán, México, a diferentes dosis. Cada punto es la media de 12 repeticiones \pm error estándar. Valores con la misma letra dentro de columnas son iguales de acuerdo con la prueba de Tukey a una $\mathrm{P} \leq 0.05$.

En la interacción especie-horas de exposición que se reporta en la Figura 2, se puede notar que las horas de exposición son determinantes para la efectividad de los polvos, lo cual resulta más notorio para O. vulgare y $O$. basilicum, donde la diferencia de mortalidad de las $24 \mathrm{~h}$ respecto a las $48 \mathrm{~h}$ fue de $50 \%$, mientras que para el epazote fue de $5 \%$. En cuanto a la interacción dosis-tiempo, está claro que ambos tienen una gran influencia para la expresión de una mayor efectividad 
en la mortalidad; como se puede apreciar, existe un efecto ascendente de acuerdo con las dosis empleadas y el tiempo de exposición (Figura 2).

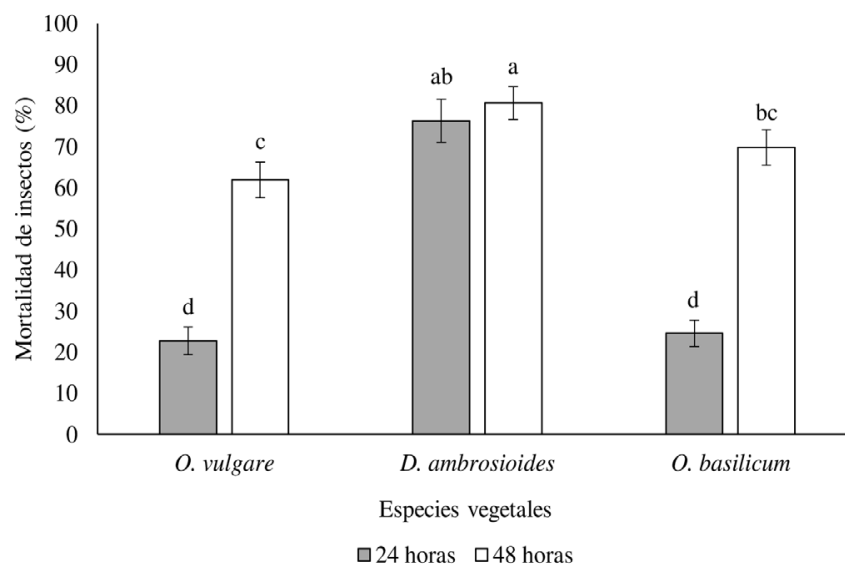

Figura 2. Mortalidad de adultos de Aethina tumida expuestos a polvos vegetales de tres especies de plantas colectadas en Yucatán, México, por periodo de 24 y 48 horas. Cada punto es la media de 12 repeticiones \pm error estándar. Valores con la misma letra dentro de columnas son iguales de acuerdo con la prueba de Tukey a una $\mathrm{P} \leq 0.05$.

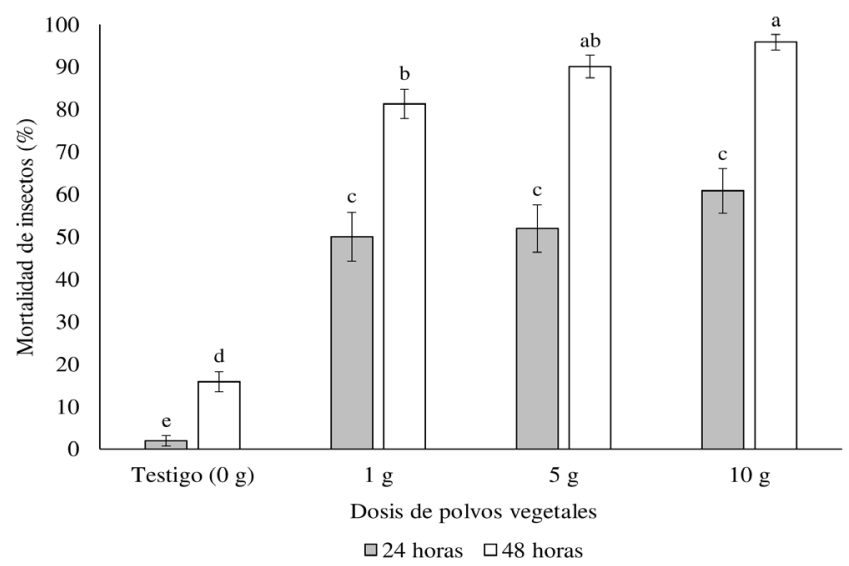

Figura 3. Mortalidad de adultos de Aethina tumida en relación con la dosis de polvo vegetal y el tiempo de exposición de tres especies de plantas. Cada punto es la media de 12 repeticiones \pm error estándar. Valores con la misma letra dentro de columnas son iguales de acuerdo con la prueba de Tukey a una $\mathrm{P} \leq 0.05$.

Estos comportamientos en las interacciones sugieren que los factores dosis-especie y especie-tiempo de exposición son determinantes para la expresión de un mayor efecto en $O$. basilicum y $O$. vulgare. Por el contrario, para D. ambrosioides, éstos fueron indiferentes al efecto, por lo que pueden apreciarse valores similares en la respuesta; lo anterior lo sugiere como el mejor tratamiento, debido a que la expresión de su efectividad puede ocurrir en menor tiempo y con la dosis baja. El poder biocida reflejado por D. ambrosioides sobre A. tumida se debe al contenido de cis-ascaridole $(60.33 \%)$, m-cimeno $(22.17 \%)$ y $\alpha$-terpinene (1.79\%), combinado con el solvente etanol (Mokni et al. 2019), ya que dichas sustancias actúan inhibiendo la acetilcolinesterasa (enzima responsable de la transmisión de los impulsos nerviosos que van desde las fibras nerviosas hasta las células musculares y glandulares), con $\mathrm{IC}_{50}$ igual a $77 \mu \mathrm{g} / \mathrm{mL}$ (Pavela et al. 2018). Estos elementos le confieren propiedades antibacterianas contra Staphylococcus aureus (256 $\mu \mathrm{g} /$ $\mathrm{mL}$ ) y Pseudomonas aeruginosa $(512 \mu \mathrm{g} / \mathrm{mL}$ ) (Bezerra et al. 2019), y poder antiviral contra el virus Coxsackie humano B (CV-B4), con $\mathrm{IC}_{50}$ igual a $21.75 \mu \mathrm{g} / \mathrm{mL}$ (Mokni et al. 2019). Además, presenta actividades antioxidantes significativas, citotóxico, antimicrobiano y antidiabético (Zohra et al. 2018).

Asimismo, la respuesta observada para el polvo vegetal a base de O. basilicum demostró efectividad de 69.2\% con la dosis de $10 \mathrm{~g}$ (Figura 3); esto concuerda con los resultados publicados por Rodríguez-González et al. (2019), quienes documentaron que la aplicación de $120 \mu \mathrm{L}$ de aceite esencial a base de O. basilicum por caja Petri (90 mm de diámetro con 20 gorgojos) presenta $74.94 \%$ de mortalidad sobre el gorgojo del frijol Acanthoscelides obtectus Say (Coleoptera: Bruchidae), además de reducir significativamente la cantidad de frijoles dañados. Similar respuesta se obtuvo sobre larvas y adultos de Tribolium confusum DuVal (Coleoptera: Tenebronidae), donde se alcanzó un control de 74 y $66 \%$, respectivamente, después de $24 \mathrm{~h}$ de exposición a $250 \mathrm{ppm}$ con el extracto de $O$. basilicum (Rodríguez-González et al. 2019). En este sentido, se ha señalado que cada $0.027 \mathrm{mg}$ de insecto por un gramo del extracto de O. basilicum causa $50 \%$ de mortalidad de la población de larvas de Spodoptera frugiperda (J. E. Smith), con un índice de toxicidad de 1194.07 (Cruz et al. 2017). Por su parte, 23.44, $21.17 \mathrm{y}$ 18.56 ppm presentan $95 \%$ de mortalidad para larvas de Culex tritaeniorhynchus Giles (Diptera: Culicidae), Aedes albopictus Skuse (Diptera: Culicidae) y Anopheles subpictus Grassi (Diptera: Culicidae), respectivamente (Govindarajan et al. 2013); estas evidencias exponen la capacidad de $O$. basilicum de generar toxicidad y repelencia significativa, lo que la convierten en una herramienta sostenible para el manejo de plagas (Silva et al. 2017); cabe señalar que la actividad insecticida de $O$. basilicum puede deberse a la presencia de linalool (52.42\%), metil eugenol (18.74\%) y 1, 8-cineol (5.61\%) en las hojas (Govindarajan et al. 2013). 
Origanum vulgare demostró mayor efectividad con la dosis de $10 \mathrm{~g}$ de polvo (65.8\%), lo cual respalda su acción tóxica en plagas. Al respecto, Salvadores et al. (2007) sostienen que $O$. vulgare presenta acción insecticida de contacto contra Sitophilus zeamais Motschulsky (Coleoptera: Curculionidae) en concentraciones de $4 \%$ (p/p), causando $80 \%$ de mortalidad; deigual manera, Baricevic et al. (2001) mencionan que $O$. vulgare posee toxicidad como fumigante y de contacto; esta última opción resulta la más efectiva para el control del gorgojo del frijol (Acanthoscelides obtectus Say), al causar $100 \%$ de mortalidad de la población cuando se aplicó de manera directa 1 y $2 \mathrm{~g}$ del polvo por $55 \mathrm{~g}$ de frijol en caja Petri. Esta actividad insecticida se debe al carvacrol (58.13\%), p-cimeno (17.85\%), timol (8.15\%), $\gamma$-terpinene $(4.96 \%)$ y linalool $(3.69 \%)$, sintetizados y acumulados por la planta (Téllez y Nolazco 2017; Xie et al. 2019). En este mismo sentido, extracto de $O$. vulgare a 12 y 15\% de concentración obtuvieron 98 y $100 \%$ de mortalidad de ninfas y adultos de Bemisia tabaci Gennadius (Vite-Vallejo et al. 2018) y con $0.25 \mu \mathrm{L}$ $/ \mathrm{cm}^{2}$ de aceite esencial de O. vulgare se reporta $90.9 \%$ de mortalidad de pupas de Musca domestica L. (Diptera: Muscidae) (Xie et al. 2019). Nasr et al. (2017) reportan efectos larvicidas (tercer estadio) con $1.528 \%$ (v/v) sobre Plutella xylostella L. (Lepidoptera: Plutellidae), mientras que Carhuallanqui et al. (2020) reportan a O. vulgare con efecto microbiano contra Listeria monocytogenes y Staphylococcus aureus.

El patrón de comportamiento concentración-tiempo de las especies estudiadas registró diferencias ( $\mathrm{P} \leq 0.05)$, lo que coincide con Santiago et al. (2014); dichos autores sostienen que a mayor tiempo de exposición $(144 \mathrm{~h})$ mayor mortalidad $(97.5 \%)$, cuando aplicaron extracto acuoso de D. ambrosioides sobre adultos de Cryptolaemus montrouzieri Mulsant (Coleoptera: Coccinellidae). En este mismo sentido, Cruz et al. (2017) sostienen que al usar aceite esencial de $O$. basilicum, la repelencia y la mortalidad de $S$. frugiperda fue en aumento conforme se aumentó la dosis $(0.05$ a $0.40 \%$ ) y el tiempo ( 1 a $4 \mathrm{~h}$ ); en otro estudio similar, mencionan que el extracto de $O$. vulgare a dosis de $4 \%$ causa $80 \%$ de mortalidad 96 h después aplicar el tratamiento sobre adultos de S. zeamais (Salvadores et al. 2007); este comportamiento quizá se deba a que $D$. ambrosioides, O. basilicum y O. vulgare actúan inhibiendo la acetilcolinesterasa de los insectos, y que, a mayor dosis y tiempo de exposición, presenta mayor efecto toxico (Pavela et al. 2018).

Estos resultados y los de otros autores afianzan la idea del uso de extractos vegetales para el control de plagas agrícolas y pecuarias de manera sustentable; esto contribuye a lo que demanda la sociedad, debido a los problemas ambientales y de salud que han ocasionado los pesticidas sintéticos. Además, se suma una plaga más dentro de la lista de insectos controlados por estas tres especies de plantas y da la pauta para seguir indagando la acción insecticida de otras especies vegetales para el control del escarabajo de la colmena (A. tumida), el cual se ha convertido en una preocupación para los apicultores.

\section{Conclusión}

Los polvos vegetales a base de epazote (Dysphania ambrosioides), albahaca (Ocimum basilicum) y orégano (Origanum vulgare) presentaron efecto tóxico para A. tumida, en comparación con el testigo. El estudio demuestra que $1 \mathrm{~g}$ de $D$. ambrosioides presenta $100 \%$ de mortalidad de los adultos de $A$. tumida después de 24 $\mathrm{h}$ de tratamiento; no así para los tratamientos a base de O. basilicum y O. vulgare, donde la dosis y el tiempo desempeñaron un papel muy importante.

\section{Agradecimientos}

Sinceros agradecimientos a Randy Najaliel González González, por su participación en el desarrollo de esta investigación; de igual forma, se le agradece al Instituto Nacional de Investigaciones Forestales, Agrícolas y Pecuarias (Centro Experimental, Mocochá, Yucatán), por facilitar las instalaciones para llevar a cabo esta investigación. 


\section{LITERATURA CITADA}

Abbott WS. 1925. A method of computing the effectiveness of an insecticide. Journal of Economic Entomology 18: 265-267. https://doi.org/10.1093/jee/18.2.265a

Al Toufailia H, Alves DA, Bená DC, Bento JMS, Iwanicki NSA, Cline AR, Ellis JD, Ratnieks FL. 2017. First record of small hive beetle Aethina tumida Murray, in South America. Journal of Apicultural Research 56: 76-80. https://doi.org/10.1080/00218839.2017.1284476

Arena JS, Omarini AB, Zunino MP, Peschiutta ML, Defagó MT, Zygadlo JA. 2018. Essential oils from Dysphania ambrosioides and Tagetes minuta enhance the toxicity of a conventional insecticide against Alphitobius diaperinus. Industrial Crops and Products 122: 190-194. https://doi. org/10.1016/j.indcrop.2018.05.077

Baba-Tierto N. 1994. The ability of powders and slurries from ten plant species to protect stored grains from attack by Prostephanus truncatus (Horn) (Coleoptera: Bostrichidae) and Sitophilus oryzae L. (Coleoptera: Curculionidae). Journal of Stored Products Research 30: 297-301. https://doi.org/10.1016/S0022-474X(94)90321-2

Baricevic C, Milevoj L, Borstnik J. 2001. Insecticidal effect of oregano (Origanum vulgare L. ssp. hirtum Ietswaart) on bean weevil (Acanthoscelides obtectus Say). International Journal of Horticultural Science 7: 84-88. https://doi. org/10.31421/IJHS/7/2/272

Bezerra JWA, Costa AR, de Freitas MA, Rodríguez FC, de Souza MA, da Silva ARP, dos Santos ATL, Linhares KV, Coutinho HDM, Silva JRL, Morais-Braga MFB. 2019. Chemical composition, antimicrobial, modulator and antioxidant activity of essential oil of Dysphania ambrosioides (L.) Mosyakin \& Clemants. Comparative Immunology, Microbiology and Infectious Diseases 65: 58-64. https://doi.org/10.1016/j.cimid.2019.04.010

Calderón RA, Ramírez M. 2019. New record of the small hive beetle, Aethina tumida, in africanized honey bee colonies in Costa Rica. Bee World 96: 87-89. https://doi.org/ 10.1080/0005772X.2019.1579294

Carhuallanqui A, Salazar ME, Ramos D. 2020. Efecto antimicrobiano del aceite esencial de Orégano frente a Listeria monocytogenes y Staphylococcus aureus. Revista de Investigaciones Altoandinas 22: 25-33. http://doi. org/10.18271/ria.2020.530

[COFEPRIS] Comisión Federal para la Protección contra Riesgos Sanitarios. [internet]. 2019. Consulta de Registros Sanitarios de Plaguicidas, Nutrientes Vegetales y LMR [cited 2020 Jul 28]. Disponible en: http://siipris03.cofepris.gob.mx/Resoluciones/Consultas/ ConWebRegPlaguicida.asp.

Cruz GS, Wanderley-Teixeira V, da Silva LM, Dutra KA, Guedes CA, de Oliveira JV, Navarro DMA, Araújo BC, Teixeira ÁAC. 2017. Chemical composition and insecticidal activity of the essential oils of Foeniculum vulgare Mill., Ocimum basilicum L., Eucalyptus staigeriana F. Muell. ex Bailey, Eucalyptus citriodora Hook and Ocimum gratissimum L. and their major components on Spodoptera frugiperda (Lepidoptera: Noctuidae). Journal of Essential Oil Bearing Plants 20: 1360-1369. https://doi.org/10.1080 /0972060X.2017.1383192

[FAO] Food and Agriculture Organization of the United Nations. [internet]. 2010. Dirección de Estadística. [cited 2020 Feb 3]. Disponible en: http://www.fao.org/faostat/ en/\#home.

Govindarajan M, Sivakumar R, Rajeswary M, Yogalakshmi K. 2013. Chemical composition and larvicidal activity of essential oil from Ocimum basilicum (L.) against Culex tritaeniorhynchus, Aedes albopictus and Anopheles subpictus (Diptera: Culicidae). Experimental Parasitology 134: 7-11. http://doi.org/10.1016/j.exppara.2013.01.018

Lundie AE. 1940. The Small Hive Beetle Aethina tumida. Department of Agriculture and Forestry, Union of South Africa. Government Printer. Pretoria, South Africa.

Magaña MÁ, Sanginés JR, Lara PE, Salazar LDL, Leyva CE. 2017. Competitividad y participación de la miel mexicana en el mercado mundial. Revista Mexicana de Ciencias Pecuarias 8: 43-52. http://doi.org/10.22319/ rmcp.v8i1.4304

May T. 2015. Apicultura y conservación de la biodiversidad en el Caribe -muchos intereses convergentes y algunos divergentes - estudio de caso: República Dominicana. Ambiente y Sostenibilidad 5: 69-77. https:// doi.org/10.25100/ays.v5i1.4303

Medina-Flores CA, Guzmán-Novoa E, Espinosa-Montaño LG, Uribe-Rubio JL, Gutiérrez-Luna R, Gutiérrez-Piña FJ. 2014. Frecuencia de varroosis y nosemosis en colonias de abejas melíferas (Apis mellifera) en el estado de Zacatecas, México. Revista Chapingo serie Ciencias Forestales y del Ambiente 20: 159-167. http://doi. org/10.5154/r.rchscfa.2013.08.028

Mokni RE, Youssef FS, Jmii H, Khmiri A, Bouazzi S, Jlassi I, Jaidane H, Dhaouadi H, Ashour ML, Hammami S. 2019. The essential oil of tunisian Dysphania ambrosioides and its antimicrobial and antiviral properties. Journal of 
Essential Oil Bearing Plants 22: 282-294. https://doi.org/1 0.1080/0972060X.2019.1588171

Mürrle T, Neumann P. 2004. Mass production of small hive beetles Aethina tumida (Coleoptera: Nitidulidae). Journal of Apicultural Research 43: 144-145. https://doi.org/10.1 080/00218839.2004.11101125

Nasr M, Sendi JJ, Moharramipour S, Zibaee A. 2017. Evaluation of Origanum vulgare L. essential oil as a source of toxicant and an inhibitor of physiological parameters in diamondback moth, Plutella xylustella L. (Lepidoptera: Pyralidae). Journal of the Saudi Society of Agricultural Sciences 16: 184-190. https://doi. org/10.1016/j.jssas.2015.06.002

Neumann P, Evans JD, Pettis JS, Pirk CW, Schäfer MO, Tanner G, Ellis JD. 2013. Standard methods for small hive beetle research. Journal of Apicultural Research 52: 1-32. https://doi.org/10.3896/IBRA.1.52.4.19

Neumann P, Pettis JS, Schäfer MO. 2016. Quo vadis Aethina tumida? Biology and control of small hive beetles. Apidologie 47: 427-466. https://doi.org/10.1007/ s13592-016-0426-x

Palmeri V, Scirtò G, Malacrinò A, Laudani F, Campolo O. 2015. A scientific note on a new pest for European honeybees: First report of small hive beetle Aethina tumida, (Coleoptera: Nitidulidae) in Italy. Apidologie 46: 527529. https://doi.org/10.1007/s13592-014-0343-9

Pavela R, Maggi F, Lupidi G, Mbuntcha H, Woguem V, Womeni HM, Barboni L, Tapondjou LA, Benelli G. 2018. Clausena anisata and Dysphania ambrosioides essential oils: from ethno-medicine to modern uses as effective insecticides. Environmental Science and Pollution Research 25: 10493-10503. https://doi.org/10.1007/s11356-017-0267-9

Pereira SN, Gottschalk S, Palmeira JLT, Paulino JM, Antunes RM, Boechat RM, da Silva VG, Morales PH, Soares LH, Prezoto F. 2019. Notes on Aethina tumida Murray (Coleoptera: Nitidulidae) in an apiary in the state of Rio de Janeiro. EntomoBrasilis 12: 88-90. http://doi. org/10.12741/ebrasilis.v12i2.824

Pirk CW, Strauss U, Yusuf AA, Démares F, Human H. 2016. Honeybee health in Africa-a review. Apidologie 47: 276300. https://doi.org/10.1007/s13592-015-0406-6

Rodríguez-González Á, Álvarez-García S, González-López Ó, Da Silva F, Casquero PA. 2019. Insecticidal properties of Ocimum basilicum and Cymbopogon winterianus against Acanthoscelides obtectus, insect pest of the common bean (Phaseolus vulgaris L.). Insects 10: 151. https:// doi.org/10.3390/insects10050151
Salvadores Y, Silva G, Tapia M, Hepp R. 2007. Polvos de especias aromáticas para el control del gorgojo del maíz, Sitophilus zeamais Motschulsky, en trigo almacenado. Agricultura Técnica (Chile) 67: 147-154. http://doi. org/10.4067/S0365-28072007000200004

Santiago V, Bonifaz E, Alegre A, Iannacone J. 2014. Toxicidad de Chenopodium ambrosioides (Amaranthaceae) e Imidacloprid sobre Cryptolaemus montrouzieri (Coleoptera: Coccinellidae). Cátedra Villarreal 2: 19-27. http://doi.org/10.24039/cv20142124

[SAS] Statistical Analysis System. 2011. SAS, Versión 9.1. Institute Inc. Cary, USA.

Silva SM, da Cunha JPAR, de Carvalho SMD, Zandonadi CHS, Martins RC, Chang R. 2017. Ocimum basilicum essential oil combined with deltamethrin to improve the management of Spodoptera frugiperda. Ciencia y Agrotecnologia 41: 665-675. http://doi. org/10.1590/1413-70542017416016317

Sosenski P, Domínguez CA. 2018. El valor de la polinización y los riesgos que enfrenta como servicio ecosistémico. Revista Mexicana de Biodiversidad 89: 961-970. http:// dx.doi.org/10.22201/ib.20078706e.2018.3.2168

Téllez LA, Nolazco DM. 2017. Estudio de la composición química del aceite esencial de orégano (Origanum vulgare spp.) de Tacna. Ingeniería Industrial 35: 195-205. http://dx.doi.org/10.26439/ing.ind2017.n035.1801

Tucuch-Haas JI, Rodríguez-Maciel JC, Lagunes-Tejeda Á, Silva-Aguayo G, Aguilar-Medel S, Robles-Bermúdez A, González-Camacho JM. 2010. Toxicidad de spiromesifen en los estados biológicos de Bactericera cockerelli (Sulc) (Hemiptera:Triozidae). Neotropical Entomology 39:436440. https://doi.org/10.1590/S1519-566X2010000300019

Vite-Vallejo O, Barajas-Fernández MG, Saavedra-Aguilar M, Cardoso-Taketa A. 2018. Insecticidal effects of ethanolic extracts of Chenopodium ambrosioides, Piper nigrum, Thymus vulgaris, and Origanum vulgare against Bemisia tabaci. Southwestern Entomologist 43: 383-393. https:// doi.org/10.3958/059.043.0209

Xie Y, Huang Q, Rao Y, Hong L, Zhang D. 2019. Efficacy of Origanum vulgare essential oil and carvacrol against the housefly, Musca domestica L. (Diptera: Muscidae). Environmental Science and Pollution Research 26: 23824-23831. https://doi.org/10.1007/s11356-019-05671-4

Zohra T, Ovais M, Khalil AT, Qasim M, Ayaz M, Shinwari ZK. 2018. Extraction optimization, total phenolic, flavonoid contents, HPLC-DAD analysis and diverse pharmacological evaluations of Dysphania ambrosioides (L.) 
Mosyakin \& Clemants. Natural Products Research 33:

136-142. https://doi.org/10.1080/14786419.2018.1437428 